\title{
BMJ Experiences of patients and Open professionals participating in the HITS home blood pressure telemonitoring trial: a qualitative study
}

\author{
Janet Hanley, ${ }^{1}$ Jenny Ure, ${ }^{1}$ Claudia Pagliari, ${ }^{2}$ Aziz Sheikh, ${ }^{2}$ Brian McKinstry ${ }^{2}$
}

To cite: Hanley J, Ure J, Pagliari C, et al. Experiences of patients and professionals participating in the HITS home blood pressure telemonitoring trial: a qualitative study. BMJ Open 2013;3: 002671 .

doi:10.1136/bmjopen-2013002671

- Prepublication history and additional material for this paper are available online. To view these files please visit the journal online (http://dx.doi.org/10.1136/ bmjopen-2013-002671).

Received 1 February 2013 Revised 14 March 2013 Accepted 8 April 2013

This final article is available for use under the terms of the Creative Commons Attribution Non-Commercial 2.0 Licence; see http://bmjopen.bmj.com

\footnotetext{
${ }^{1}$ School of Nursing, Midwifery and Social Care, Edinburgh Napier University, Edinburgh, UK Sciences, The University of Edinburgh, Edinburgh, UK

Correspondence to Dr Janet Hanley; j.hanley@napier.ac.uk
} ${ }^{2}$ Centre for Population Health

\begin{abstract}
Objectives: To explore the experiences of patients and professionals taking part in a randomised controlled trial (RCT) of remote blood pressure (BP) telemonitoring supported by primary care. To identify factors facilitating or hindering the effectiveness of the intervention and those likely to influence its potential translation to routine practice.
\end{abstract}

Design: Qualitative study adopting a qualitative descriptive approach.

Participants: 25 patients, 11 nurses and 9 doctors who were participating in an RCT of BP telemonitoring. A maximum variation sample of patients from within the trial based on age, sex and deprivation status of the practice was sought.

Setting: 6 primary care practices in Scotland.

Method: Data were collected via taped semistructured interviews. Initial thematic analysis was inductive. Multiple strategies were employed to ensure that the analysis was credible and trustworthy.

Results: Prior to the trial, both patients and professionals were reluctant to increase the medication based on single BP measurements taken in the surgery. BP measurements based on multiple electronic readings were perceived as more accurate as a basis for action. Patients using telemonitoring became more engaged in the clinical management of their condition. Professionals reported that telemonitoring challenged existing roles and work practices and increased workload. Lack of integration of telemonitoring data with the electronic health record was perceived as a drawback.

Conclusions: BP telemonitoring in a usual care setting can provide a trusted basis for medication management and improved BP control. It increases patients' engagement in the management of their condition, but supporting telemetry and greater patient engagement can increase professional workloads and demand changes in service organisation. Successful service design in practice would have to take account of how additional roles and responsibilities could be realigned with existing work and data management practices. The embedded qualitative study was included in the protocol for the HITS trial registered with ISRCTN no. 72614272.

\section{ARTICLE SUMMARY}

\begin{abstract}
Article focus
- Qualitative exploration of the experiences of patients and professionals taking part in a randomised controlled trial (RCT) of remote blood pressure (BP) telemonitoring supported by primary care.

- Identification of factors facilitating or hindering the success of the intervention and those likely to influence its potential translation to routine practice.
\end{abstract}

\section{Key messages}

- BP telemonitoring in a usual care setting can provide a trusted basis for medication management and improved BP control.

- It increases patients' engagement in the management of their condition.

- Supporting telemonitoring and greater patient engagement can increase professional workloads and demand changes in service organisation.

Strengths and limitations of this study

- The strengths of the study are that it is based on experience of using the systems by the patients' own practitioners in a usual care context.

- The trial context permitted triangulation with quantitative data. Owing to the protocolpermitted evolution in practice, it gives an indication of some of the issues which would need to be addressed for BP telemonitoring to be used in routine practice.

- The weaknesses are that the participation in the study was relatively short for each practice with limited patient numbers, so any longer term barriers to evolution in practice were not identified. It is also possible that participants in this study differ from non-participants.

\section{BACKGROUND}

Long-term illness is increasingly prevalent, and telemonitoring (remote self-monitoring of health parameters with electronic transmission of data to a healthcare provider) is considered to be a promising way of 
supporting patient care within existing resources. ${ }^{1}$ However, despite policy statements and numerous pilots, telemonitoring has not yet been widely adopted. ${ }^{2}$ This qualitative study examined patient and professional experiences of blood pressure (BP) telemonitoring in the context of a randomised controlled trial (RCT). As an embedded qualitative study, its purpose was to help explain the trial results and to generate insights regarding factors likely to influence the adoption of this approach within routine care.

Hypertension (persistently raised BP >140/ $90 \mathrm{~mm} \mathrm{Hg}$ ) is a major cardiovascular risk factor, which is frequently poorly controlled, ${ }^{3}$ with evidence of under treatment in many cases, sometimes described as "therapeutic inertia. ${ }^{4}$ This is despite the availability of guidelines ${ }^{5}{ }^{6}$ or (as in the UK) financial incentives to primary care doctors. ${ }^{7}$ In day-to-day practice, effective assessment of BP is problematic. Single BP measurements taken in the surgery are poorer indicators of risk than estimates based on multiple measures from ambulatory or home monitoring, ${ }^{8-10}$ and 'white coat hypertension'-raised $\mathrm{BP}$ when measured in the surgery, but not at home, ${ }^{11}$ is a complicating factor. However, for practical reasons, surgery-based measurements are still the basis of treatment decision-making in most cases. Telemonitoring can overcome these measurement issues by allowing patients to take multiple BP readings at home and share them with healthcare professionals in almost real time, potentially providing motivation for improvements in self-care while facilitating professional input if necessary. Although we have been unable to identify previous qualitative studies of telemonitoring in hypertension in a usual care setting, some common themes are emerging from qualitative studies where the self-monitoring was either part of a larger intervention such as specialist nursing support or self-management of medication, ${ }^{12} 13$ or the patients were simply self-monitoring with no data transmission. ${ }^{14}{ }^{15}$ The common themes were that patients generally find self-monitoring to be a positive experience which is empowering, reassuring and motivational.

The trial which formed the context for this study ${ }^{16}$ (see box 1 for summary of the intervention) involved patients from primary care hypertension registers whose surgery BP measures in the previous 6 months had been $>140 / 90 \mathrm{~mm} \mathrm{Hg}$, who had a BP higher than 135/ $85 \mathrm{~mm} \mathrm{Hg}$ on daytime ambulatory BP monitoring performed as screening for the trial and did not have diabetes, previous stroke or transient ischaemic attack, atrial fibrillation or other major illness. It found that for those using telemonitoring, BP reduced by a mean of 4.3/2.6 $\mathrm{mm} \mathrm{Hg}$ compared with the group receiving usual care. Other trials in this field also strongly suggest that telemonitoring in hypertension can be effective in achieving clinically important reductions in systolic and diastolic $\mathrm{BP},{ }^{17}{ }^{18}$ but some studies have shown poorer outcomes. ${ }^{19}$ The introduction of telemonitoring may be regarded as a complex sociotechnical intervention involving changes in behaviour in addition to a purely technological solution. It is therefore important to understand how the components of intervention and contextual factors contribute to the outcome. ${ }^{20}$ These issues can be difficult to explore using quantitative methods alone ${ }^{21}$ and proponents of 'realist evaluation' suggest using qualitative methods to tease out what works in different contexts. ${ }^{22}$ The aim of this study, therefore, was to qualitatively explore the experiences of patients and professionals taking part in a trial of BP telemonitoring based in a usual care setting, to identify what contributed to the effectiveness of the intervention, what limited its effectiveness and what may be required for the success of the trial to be translated into routine care.

\section{METHODS}

\section{Overview}

This study was embedded within an RCT of BP telemonitoring in routine care for patients whose $\mathrm{BP}$ was above target $^{16}$ (box 1). A qualitative descriptive approach was employed, ${ }^{23}$ acknowledging that in health services research the need of the researcher is not only simply provide a description of the phenomenon, but also to produce an interpretive account which will help to guide healthcare innovation while, at the same time, recognising the subjective nature of the encounter between the subject and the researcher. ${ }^{24}$

\section{Ethics and governance considerations}

The study received ethical approval from the South East Scotland Ethics Service (08/S1101/38) and research and development approval from NHS Lothian. Patients were made aware that they may be approached for the embedded qualitative study when they agreed to participate in the trial, but that participation in this study would be optional. Patients and professionals approached were sent a separate information sheet about the qualitative study and signed an additional consent prior to participation.

\section{Sampling and recruitment}

Twenty general practitioner (GP) practices and 401 patients participated in the RCT. Of these, patients and staff from five socioeconomically diverse practices (based on the Scottish Index of Multiple Deprivation ${ }^{25}$ ) were initially approached to participate in the qualitative study. A sixth practice was added later to increase the number of professionals participating and ensure data saturation. A maximum variation patient sample of at least 20 patients overall from these practices was sought based on age, sex and the deprivation status of the practice. Patients participating in the trial were purposively sampled and checks were made with the practice to ensure that it was still appropriate to approach the patient before they were contacted by letter. Those who did not respond were replaced by patients with similar 


\section{Box 1 Description of the telemonitoring intervention}

\section{The intervention}

The practices and patients were asked to use a system which comprised a validated electronic home blood pressure (BP) monitor and mobile phone technology that enabled the transfer of BP readings via SMS to a secure website which was accessible to the user and their doctor or nurse, and also provided automated feedback to the patient. The BP monitor linked to a mobile phone wirelessly, via Bluetooth. The components of the intervention were

- Home BP monitoring: Patients were asked to record their BP as agreed with the healthcare team, or more frequently as they wished. Guidance was initially to record BP twice in the morning and twice in the evening for a week in line with the European guideline on BP monitoring, to build a baseline average. Thereafter, they were asked to take weekly measurements preferably at different times of the day if their average BP was within the recommended range; however, if they had made any lifestyle or medication change which would impact on their BP, they were asked to measure their BP for a more intensive period of monitoring to allow the rolling average to change and to more quickly assess the effect.

- Transmission of data: This simply required the phone to be switched on and to have a signal when the BP measurement was taken. Patients just had to apply the cuff and press a button on the BP monitor. The reading and transmission occurred automatically. Mobile phone problems did not lead to loss of data because all readings were stored in the monitor and any untransmitted readings were sent when the next reading was taken.

- Feedback to patients (closed loop feedback): In addition to optionally accessing their BP record on-line, patients could also opt to receive reports via text message or email. These gave advice on the current status of their BP based on the average of the last 10 readings, and whether they should contact their doctor or nurse. Reports were generated every 10 readings or weekly, whichever was sooner, with a reminder to check BP if this had not been done. These reports could reassure them that their average BP was within target $(<135 /$ $85 \mathrm{~mm} \mathrm{Hg}$ ) or tell them that their BP average had improved on the last report but had not attained the target and to maintain current therapy, or that their BP was not at target and that they should contact their clinician. If an individual BP reading was very high (>220/ $120 \mathrm{~mm} \mathrm{Hg}$ ), an immediate text or email report was generated reinforcing the written advice in the patient information leaflet to rest for 30 min, check again and contact the practice if BP remained very high.

- Sharing the readings with the healthcare team: Members of the healthcare team were able to access the records of their patients online via a secure login to a summary screen which listed their patients, their average BP over the last 10 readings, and the date of their last reading. Average BPs outside the recommended limits (set at $135 / 85 \mathrm{~mm} \mathrm{Hg}$ for the study) were highlighted. Clicking on the each individual patient led to lists or graphs of all their readings. Clinicians could then check the patients' electronic general practitioner (GP) record to see if there had been recent advice regarding medication or lifestyle change and if not, could contact the patient to make a change. Clinicians were recommended to check the website weekly, but the frequency of log-on could be chosen by them.

\section{Usual care}

Patients allocated to the usual care group were asked to continue to attend the practice for BP checks according to the usual routine of the practice. If they were already home monitoring, they were not discouraged from continuing.

\section{All patients}

For all patients, the GP/practice nurse was informed that the ambulatory monitoring used to screen for eligibility for the HITS trial had shown that their average BP was above the target range, but they were not given the actual reading. All patients were given an information pack containing a range of publicly available leaflets on hypertension management and lifestyle modification.

characteristics. The aim of this sampling strategy was to capture a broad range of patient experiences across the socioeconomic spectrum included within the trial.

\section{Data generation and handling}

Qualitative data were gathered through semistructured interviews with patients, nurses and doctors. The initial topic guides were based on issues identified by our previous acceptability study ${ }^{26}$ and interviews with patients participating in the trial pilot study. The interview topic guides were refined iteratively in response to the initial interviews. The final topic guides are shown in online supplementary appendix 1 . Most patients were interviewed face-to-face in their own home, and professionals at their workplace, with interviews carried out by telephone where this was not possible. Most healthcare professionals were interviewed individually, but two nurses were interviewed together, as were three doctors. Interviews were carried out by $\mathrm{JU}$, an experienced female qualitative researcher, with a background in education and psychology who was not involved in the RCT.

\section{Data handling and analysis}

The data were collected between July 2009 and June 2010 and, with a little variation due to availability, in tranches reflecting different start dates of different practices. Provisional coding and identification of themes took place after each tranche of interviews. Interviewing continued until the researcher, in discussion with the wider research team, considered that data saturation was achieved. Although there is a discussion on the concept of data saturation, in the context of this study which was focused and involved a relatively homogeneous population, data saturation was considered to have occurred when the researcher was not identifying any new themes or codes within the provisional themes in sequential interviews, and thought this would be unlikely in subsequent interviews. Detailed recoding and checking took 
place from May to December 2010 with the validation focus group taking place in May 2011.

All interviews were recorded, fully transcribed and the transcript checked against the recording. They were analysed thematically with initial codes and themes identified inductively from the data. Patient and professional data were coded separately. Coding was marked on the transcripts using the comments facility in Microsoft Word and the text associated with each code stored on an Excel spreadsheet.

A range of strategies was employed to ensure that the analysis was credible and trustworthy. Constant comparison was used to ensure consistency in coding and negative cases were sought for each coding category. Coding was checked and iteratively refined using paired analysis of transcripts by two researchers. Researcher reflexivity was supported by discussing emerging findings with a wider research group where different explanations were explored and the coding and thematic analysis were reviewed and refined. Following this, the thematic analysis was presented by $\mathrm{JH}$ to a discussion group of 21 patients, professionals and researchers who had participated. The presentation introduced the themes and illustrative quotes and the whole dataset (all the text associated with each code) was made available to the participants. This discussion, which lasted for $90 \mathrm{~min}$, was moderated by BM, recorded, transcribed and coded. It was used not only to validate the initial grouping of data into codes and themes but also to extend the discussion of how telehealth may change the provision of primary care. The coding is shown in online supplementary appendix 2 .

The themes were grouped into four overarching themes presented here. The groupings were broadly informed by the purpose of the study and also by the normalisation process model as applied to telehealth, with its constructs of interactional workability, relational integration, skill set workability and contextual integration. ${ }^{27}$ Online supplementary appendix 2 shows the codes (with the number of text extracts coded against each) and how they have been grouped into themes and overarching themes. Where it was possible to triangulate findings arising from the qualitative data against the quantitative trial data (including workload impacts, lifestyle change and the impact of telemonitoring compared with simple home monitoring), this was also done. Three overarching themes are presented here. The fourth theme comprised comments about the study and is shown in online supplementary appendix 2 .

\section{RESULTS}

Thirty-four patients were approached (28 from the intervention arm and 8 from the control arm) and 25 patients ( 5 from the control arm of the trial) participated. The patients' characteristics are shown in table 1 . Eleven practice nurses (all women) and nine GPs (4 men,

\begin{tabular}{|c|c|c|c|c|c|c|c|c|c|c|}
\hline \multirow[b]{2}{*}{ Patient } & \multicolumn{2}{|c|}{ Sex } & \multicolumn{3}{|l|}{ Age } & \multicolumn{2}{|l|}{ Group } & \multicolumn{3}{|c|}{ Deprivation level of practice } \\
\hline & $\overline{\mathbf{M}}$ & $\bar{F}$ & $<50$ & $50-9$ & $\overline{70+}$ & Monitoring & Not monitoring & Least deprived & Mixed & Deprived \\
\hline 1 & & $x$ & & & $x$ & & $x$ & & & $x$ \\
\hline 2 & $x$ & & $X$ & & & $X$ & & & $x$ & \\
\hline 3 & $x$ & & & $x$ & & $x$ & & & & $x$ \\
\hline 4 & $x$ & & & $x$ & & & $x$ & & $x$ & \\
\hline 5 & $x$ & & & & $x$ & $x$ & & & & $X$ \\
\hline 6 & $x$ & & & $X$ & & $X$ & & & $X$ & \\
\hline 7 & & $x$ & & $X$ & & $x$ & & & & $X$ \\
\hline 8 & $x$ & & & $x$ & & & $x$ & & $X$ & \\
\hline 9 & $x$ & & $X$ & & & & $x$ & & & $X$ \\
\hline 10 & & $x$ & & $X$ & & $X$ & & & $X$ & \\
\hline 11 & $x$ & & & & $x$ & $x$ & & $x$ & & \\
\hline 12 & $x$ & & & $x$ & & $x$ & & & $x$ & \\
\hline 13 & $x$ & & & $x$ & & $x$ & & $x$ & & \\
\hline 14 & & $x$ & & & $x$ & $X$ & & & $x$ & \\
\hline 15 & & $x$ & & & $x$ & $x$ & & $x$ & & \\
\hline 16 & & $x$ & & $X$ & & $X$ & & & $x$ & \\
\hline 17 & $x$ & & & & $x$ & $x$ & & $x$ & & \\
\hline 18 & & $x$ & & & $X$ & $X$ & & $X$ & & \\
\hline 19 & $x$ & & & & $x$ & $x$ & & $x$ & & \\
\hline 20 & $x$ & & $X$ & & & $X$ & & $X$ & & \\
\hline 21 & & $x$ & & & $x$ & & $x$ & $x$ & & \\
\hline 22 & $x$ & & $X$ & & & $X$ & & $X$ & & \\
\hline 23 & & $x$ & $x$ & & & $x$ & & & $x$ & \\
\hline 24 & & $x$ & & & $X$ & $X$ & & $x$ & & \\
\hline 25 & $x$ & & & $x$ & & $x$ & & & & $x$ \\
\hline
\end{tabular}


5 women) took part. Five patients from the control group were interviewed because it was possible that their treatment may have been different than usual during the trial. There was no evidence from the interviews that this had happened. The telemonitoring service employed in this study was novel in that self-monitoring was integrated with usual care. For this reason we present the patient and professional data together here, highlighting the areas of concordance, divergence and evolution in practice both between and within the professional and patient groups.

\section{Patient experience}

Patients' accounts of their response to the initial diagnosis of hypertension differed, as did their level of concern and their personal approaches to self-management. Some were not concerned, did not think of their hypertension often and left the management to their doctor or nurse. For others, the diagnosis had caused practical problems (eg, in taking out life insurance) or anxiety, particularly where they had an experience of a family member suffering a stroke. Anxiety about what was happening to their BP between appointments had led some to self-monitor their BP prior to this study, and one practice provided patients with a home monitor to use during diagnosis. Contrasting perspectives are illustrated in the quotations below:

Oh I just take my tablet. I don't think about it...I'm not the worrying kind ...I don't see the point in worrying over things.

(Patient 1, control group, previous experience of home monitoring with practice monitor)

And I'm conscious of it because what I'm looking to do you do have to have a medical, and blood pressure is one of the key things that they don't want, if you have high blood pressure you're out. So I'm looking to get it down

(Patient 20, monitoring group, no previous experience of home monitoring)

I can't remember if they...if I was advised to go and buy a home monitoring machine but I decided to do it anyway...I knew that my blood pressure would be checked every time, regularly at the surgery but certainly twice a year,... but until that I would like more information than that.

(Patient 4, control group, previous experience of home monitoring with own monitor)

The differing levels of patient concern about hypertension at the start of the study is clearly a factor which could influence outcomes, but was not something which was directly measured in the trial. ${ }^{16}$

Patients saw hypertension largely as a lifestyle issue and many tried to ascribe a cause within their lifestyle such as reduced physical activity and stress, although some also mentioned familial tendency to high BP.
I wasn't say like grossly overweight, I wasn't...didn't smoke at all, it was difficult to...I mean I did have a more difficult lifestyle at the time, a lot of commuting, a lot of driving

(Patient 6, monitoring group, no previous experience of home monitoring)

Most were aware that lifestyle change could help control BP and had been given advice. Lifestyle advice was received from multiple sources and perceived to be general rather than being targeted at the reasons for them individually developing hypertension.

...a proper balanced diet and not too much fat and all these sort of things. But it's strange if anyone doesn't know about that nowadays

(Patient 17, monitoring group, no previous experience of home monitoring)

Some participants had modified their lifestyle prior to the trial in response to the diagnosis of hypertension

What I did do [when diagnosed with hypertension] and I've stuck to it, I've cut out salt. I was overweight a few years ago and I cut out butter, so now I don't have butter and I don't have salt. (I) just (use) general knowledge, just tried to reduce salt, reduce weight. And salt brings up your blood pressure so...

(Patient 12 monitoring group, no previous experience of home monitoring)

Some of the patients interviewed had modified their lifestyle during the trial, and considered that the system provided motivation

...I had lost a stone in weight during the course of that six months as well that I was being on the monitor and I think that did. I think that was a contributory factor to my BP balancing out

(Patient 7, monitoring group, previous experience of home monitoring with practice monitor)

When I was taking the blood pressure I couldn't bear looking at a hundred and forty, a hundred and fifty over a hundred and ten and I wanted to just be able to see better readings in a way. So over the summer as well, starting to get more walking exercise, that kind of thing.... I didn't want beta blockers because they had various side effects which...I kind of felt the conventional medicine options were maybe a bit limited in terms of what I wanted out of it or in terms of avoiding side effects, so it did spur me on to look for alternative...

(Patient 2, monitoring group, no previous experience of home monitoring)

So I like having the machine there because it prompts me, and I've done things like I've done some exercise and then I've taken my blood pressure to see whether it 
has made an impact, and it did, it does, every time. (Patient 20 monitoring group, no previous experience of home monitoring)

However, many others were aware of the need for lifestyle change, but found it too difficult to implement.

...obviously I'm overweight, I'm trying to do something about that but it just doesn't happen. (Patient 4, control group, previous experience of home monitoring with own monitor)

The quantitative results of the trial ${ }^{16}$ did not show any significant changes to lifestyle variables relating to diet, exercise or medication adherence in either the intervention or control groups. However, a closer examination of the trial data did show that in both groups a small number of individuals (10 overall) had, as described by patient 7 , lost more than 1 stone $(6.5 \mathrm{~kg})$ in weight.

Using the system increased some patients' engagement with the medical management of their hypertension. They used it to negotiate treatment and, in a very small number of cases, titrate their own medication.

It's certainly given me more meaningful data to speak to the doctor rather than, "Well, I think my BP has probably gone up."

(Patient 6, monitoring group, no previous experience of home monitoring)

...I've got 16 milligrams and eight milligrams [tablets of antihypertensive drug] and the last time I saw Dr B he said; the maximum you can have is 32 . Well what I probably could do is go and see the nurse and say can I take it upon myself to move it up to 32 by taking another eight

(Patient 19, monitoring group, no previous experience of home monitoring)

These data suggest that the interaction between patient and doctor or nurse facilitated by the telemetry is important rather than just the home monitoring. This is supported by trial data where it was possible to compare outcomes between the telemonitoring group and control group for the $30 \%$ of patients who had selfmonitored prior to the trial (this analysis was not included in the published trial results). Within this group, those randomised to telemonitoring had a mean reduction in systolic daytime ambulatory BP of $7.16 \mathrm{~mm} \mathrm{Hg}$ (95\% CI 3.67 to $10.64 \mathrm{~mm} \mathrm{Hg}$ ) compared with those receiving usual care.

Generally, both patients and professionals thought that the increased patient engagement in BP management was beneficial.

Yes. I really thought that it (system) was a brilliant idea. And it has helped me a lot, to understand more
(Patient 6, monitoring group, no previous experience of home monitoring)

...the positive thing about it was it definitely helps patients to become much more involved in their care which is a good thing and they definitely take much more interest in it I think because they're measuring it, they can see it, you know it's much more real to them I think

(Practice Nurse 9)

However, a note of caution was sounded, again by both patients and professionals, that for a small number of people home monitoring could provoke anxiety

I felt it was intrusive. I started worrying about my BP

(Patient 12 monitoring group, no previous experience of home monitoring)

....and then you've got others; 'Oh, I feel a bit ill today, I'll better check my BP'... and I think that that's the danger of home monitoring, I think people can become obsessed with it...

(Practice Nurse 6)

Although both patients and professionals raised the issue of anxiety generated by home monitoring, it was a much stronger theme among the professionals. It was only raised by one patient during the interviews, but several professionals. This accords with our previous telehealth work where there is considerable concern among professional about the possibility of telehealth putting patients in a 'sick role' and making them dependent or anxious, but this concern is not widely echoed by patients. $^{28} 29$

\section{Using the telemonitoring system}

The simple telemonitoring system used in this study generally worked well, although some design issues were highlighted as described below. Generally, patients had little difficulty measuring their BP, transmitting their readings or finding a routine for measuring their BP. Some also accessed the on-line record of their readings. However, the wording of the automated feedback messages sent by the system to was not found to be valuable although the messages in themselves did remind some patients to maintain their engagement with the system.

it's the same message from presumably a machine [laughs] which doesn't help a lot. Because it's obviously coming from a machine and it tells me have I contacted my medical practice or nurse, which I have but it doesn't seem to know that, you see?

(Patient 9, monitoring group, previous experience of home monitoring with practice monitor) 
The main clinical advantage provided by the system was that it facilitated the management of $\mathrm{BP}$ using average $\mathrm{BP}$, based on multiple readings taken at home, which was seen by both patients and professionals as a more trustworthy basis for action than single BP measurements taken in the surgery. Both patients and professionals commented that, prior to the system being introduced, there were sometimes long delays in initiating appropriate treatment in people with less markedly raised BP, driven by reluctance by healthcare staff to prescribe medication which may not be absolutely necessary. The data from the patients who were not in the intervention group suggested that, for them, medication changes remained infrequent and this was supported by the trial data. ${ }^{16}$

[prior to the trial] ... you have a BP maybe 148 over 88, you might say 'let's see you again in six months', when actually it's too high"

(Practice Nurse 10)

[prior to the trial] ...We're all guilty of it...'we'll just see how it goes, you know, maybe watch it. I'll check it again tomorrow' and they probably maybe sit on it a bit longer than they...it's just a natural thing isn't it (Practice Nurse 9)

....and this patient in particular has been quite reluctant to increase the medication because of her belief that her high BP is just a temporary thing because of what's going on her life...she doesn't think that she should be on medication at all (Practice Nurse 11)

There was consensus between both patients and professionals that the home monitoring system provided a more accurate assessment of BP than surgery measurements and better evidence for action, facilitating rapid tailoring of medication.

you're getting a more accurate insight into true BP readings (Practice Nurse 6)

Well I tried everything. I had it on the table, I had my arm on a pillow and I was trying to relax as much as I could, but there's no way you're going to cheat the machine so it's... it is a good thing like. You cannot kid yourself on with it (Patient 6, monitoring group, no previous experience of home monitoring)

One consequence of the professionals now feeling that they had an ongoing accurate estimate of the patient's average BP was to raise new questions about what to do if it BP was near, but not at, the target.

The only problem I had with it in a way is these people that were coming up as uncontrolled who were one millimetre above the control level. And I just thought, oh come on, are you really going to add in another drug to bring this down from 81 to 80 ? (GP1)
For healthcare staff, the main practical issue with the system was the lack of integration of the BP data with the main patient electronic records and the fact that not all members of the healthcare team regularly accessed the online system and were able to see the patientrecorded readings. This caused problems when patients consulted with other members of the team.

...So they're coming in to see the doctor, the doctor takes their BP, one forty five ninety; 'oh, that's fine, what are you worried about?' ... And then you go and you look at it the next week and you think; they've seen the doctor and yet their BP's still really high

(Practice Nurse 11)

The trial thus flagged wider implementation issues such as the need for reconfiguration of work practices to accommodate new roles, and ensure synergies across a more distributed care team, as well as more integrated access to patient data from disparate data sources.

\section{Adjusting to new responsibilities and new ways of working}

The new service and increased patient engagement challenged organisation within practices. Professionals reported that monitoring the electronic data increased patient contact and the need for more rapid decisionmaking raised workloads. The consensus was that a nurse-led service would be the best model, but monitoring the system needed to be an acknowledged part of the practice nurses' role with regular formal time set aside for an electronic clinic. Not all practices succeeded in monitoring the electronic data regularly throughout the trial. The trial data confirmed the increased workload with patients in the telemonitoring group having, on average, two additional consultations with the practice (one with the GP and one with the practice nurse, half of which were by telephone) over the 6 months of the trial compared with the control group. ${ }^{16}$

Care had traditionally been face-to-face, but continuing to use this model to try to respond quickly to the telemetry data had its frustrations.

We would phone them and say your BP's up, we need to increase your medication or you need to come in and see someone ... And they never made appointments and then we had to phone them again and say 'You've still not made an appointment, are you coming in?

\section{(Practice Nurse 3)}

Some professionals began to change how they worked, reducing reliance on face-to-face contact with the patients. Telephone contact increased although it was also found to be a time consuming way of reaching patients who were frequently not available during working hours.

I feel like I'm phoning these patients all the time. If they can't get them in and you'rehaving to leave a message then you're going to have to leave a message in the book 
to make sure they've got the message. You're checking to see if they've picked up a prescription because you're then checking their blood pressure-it's still high-'Have they taken their medication?' You're phoning them...

\section{(Practice Nurse 4)}

There was some experimentation in using email with appropriate safeguards and, although only a small number were involved, this worked well.

...I got an email from the doctor every week from the readings and he was able to advise me without me having to visit the doctor.

(Researcher...how did you feel about that?)

Well I was quite happy with that. I thought that was great. It was very time saving for me.

(Researcher: Ahah. So you find the time saving aspect then very useful?)

Oh, yes, yes definitely. No effort making appointments, you just got an automatic email every week. They give suggestions and like 'Take more of the tablet's. 'Take less of the tablets" and that's how it went until I got a good reading regularly, good readings

(Patient 7, monitoring group, previous experience of home monitoring with practice monitor)

Some patients also changed the way they accessed services because they now knew what they wanted, for example, bypassing the practice nurses and going directly to the GP because they thought the nurse could not prescribe a change in medication.

When the data were presented for validation to a discussion group of patients, professionals and researchers, it is this theme, of adapting to new roles and responsibilities, which dominated the discussion. Echoing the themes presented above, patients in the discussion group emphasised the role of telemetry-enabled home monitoring as being motivating, an incentive to improve self-care and evidence which facilitated meaningful conversation and dialogue with professionals. However, despite having been presented with the qualitative data on increased professional workloads and contact with patients (the figures from the trial were not available at that point), they thought that in the longer term home monitoring should lead to a reduction in the need for surgery attendance which would be appreciated by patients. The need for clarity in roles (what the patient was expected to do and what the practice was expected to do) was emphasised.

\section{DISCUSSION}

Both patients and clinicians participating in this study considered that a measurement based on the average of multiple readings from the home monitoring system was trustworthy and could be used as a basis for action. Although patients generally saw hypertension as a lifestyle issue and were aware of lifestyle interventions, only a few achieved significant lifestyle changes during the trial and some were from the control group. However, the system was described by patients as a motivator to achieve BP control and the instantly available, shared and trusted reading facilitated more rapid tailoring of medication. This was partially driven by patients who increased their engagement with the medical management of their condition, initiating contacts and negotiating treatment, and partially by the healthcare professionals who contacted people with unsatisfactory readings. The cost to the practices of achieving improved BP control was increased patient contacts and workloads for professionals during the trial (where all participants had uncontrolled BP at the start). However, when this outcome was discussed with patients and professionals, they expressed the view that in the longer term the system could reduce the need for surgery visits. There was an example of this where one patient described asynchronous communication with the practice in the form of email which resulted in tailoring of medication without the need for frequent phone calls and surgery visits.

Prior to the intervention, patients differed in their levels of concern about their hypertension and there was an acknowledgement that not all patients would respond to telemonitoring in the same way. Some clinicians were concerned that, for a small number of people, monitoring and increased engagement in the medical management of their hypertension may have provoked anxiety or dependency. This was echoed by one patient although generally increased engagement was seen to be a positive change by both patients and clinicians.

Within the practices, doctors and nurses found that traditional ways of working, which prioritises face-to-face consultations and where there may be split responsibilities for BP monitoring and antihypertensive prescribing, may not easily support the increased patient engagement or rapid treatment adjustment that successful telemonitoring requires and there was some evolution of working practices during the study. A lack of integration of telemonitoring data with the patients' electronic records also limited multidisciplinary working within the practices. The acceptance by professionals that they had an accurate estimate of the patients' BP raised questions about the best management when BP was near the recommended level which was not covered in the guidelines available at the time.

The strengths of the study are that it is based on real experience of using the systems and, because of the protocol-permitted evolution in practice, it gives an indication of some of the issues which would need to be addressed for BP telemonitoring to be used in routine practice. These include integrating of telemonitoring data with the electronic patient record, enabling communication channels between patients and professionals 
which are rapid and efficient for both, implementing interprofessional working practices which support rapid tailoring of medication and additional clinical guidance. The review by Mair et $a \vec{l}^{30}$ on factors affecting the success of telehealth implementations chimes with many of these, as do earlier qualitative studies such as May et $a \vec{l}^{11}$ Feedback from participants and triangulation with trial data add weight to these interpretations. However, practices were only involved in the trial for a short period (about 8 months) and evolution of practice to meet the needs of patients who are telemonitoring could be limited by factors which have not yet been identified. It is also possible that both patients and practices who agree to participate in a trial may differ in some way from those who choose not to participate, and thus not all opinions about telemonitoring may have been captured. A request to seek opinions about telemonitoring from those who did not want to participate in the trial was declined by the Ethics Committee. A weakness of the study was that the triangulation data showing the effectiveness of the intervention among those who already owned a home monitor were only received after the end of data collection and the opportunity was lost to interview more of this group and more systematically investigate what they considered that telehealth added to their home monitoring.

No direct comparators to this qualitative study, which examined telemonitoring provided in a usual care context, have been found. The HITS triall ${ }^{16}$ which was the context for this study, showed that patients using telemonitoring in their usual primary care setting had a greater reduction in $\mathrm{BP}$ than the control group. Patients found the equipment easy to use and the measurements easy to understand. This was similar to the experience reported in other studies with effective interventions incorporating telemonitoring such as TASMINH $2,{ }^{13}$ and differed from a recent US-based study where difficulties in using the system were reported ${ }^{32}$ and the effect was much smaller. ${ }^{18}$ System design (hardware, software and the associated guidance and support) clearly has a part to play, and providing the service via the patient's usual practitioners, rather than as a separate stand-alone service, may have also helped in terms of patient support. There is also a possibility that some of the usability issues may be related to the populations involved. In the US study, issues with poor literacy were identified, ${ }^{28}$ whereas, although the population for the trial which underpinned this study ${ }^{16}$ was drawn from across the spectrum of social privilege and deprivation in Scotland, this was not a concern raised at any point. In some of the trials of larger interventions incorporating BP telemonitoring, ${ }^{12} 1718$ it is hard to unravel the impact of the telemonitoring from the impact of the rest of the intervention. This study, and the trial outcome, suggests that the telemonitoring itself can overcome some of the barriers to an improved BP control. The added value of the additional interventions such as pharmacist support ${ }^{12}$ or medication self-management plans ${ }^{17}$ needs to be determined. Interestingly, the qualitative study associated with a trial which included telemonitoring and medication self-management suggested that although many participants would be happy to continue with the telemonitoring, few would be happy to continue with the medication self-management plans. ${ }^{13}$

The consensus among the professionals interviewed was that a nurse-led monitoring service would be most appropriate, but some patients perceived that nurses could not prescribe the changes to medication which the patients thought they needed. This could be a limiting factor for the efficiency of the service. The legislative and training framework for independent nurse prescribing has been established in Scotland, ${ }^{33}$ but the number of nurse prescribers is still very limited, although growing. ${ }^{34}$ Increasing nurse prescribing in long-term conditions may be a key to providing the organisational infrastructure to maximise the efficiency of this model of telemonitoring. Integration of the telemonitoring data with electronic patient records would also be essential; patients are free to consult with other members of the primary health care team and expect their BP data to be available. Further consideration needs to be given to the workload issues for the practices involved. The trial did increase their workloads, ${ }^{16}$ but the discussion group considered that telemonitoring could reduce the need for practice visits in the longer term. This raises the question of whether telemonitoring should be a short-term or long-term intervention. A model where initial professional surveillance of BP gives way to patient self-monitoring once control is established should be investigated.

In conclusion, this qualitative study indicates that in a UK context BP telemonitoring in a usual care setting can provide a trusted basis for medication management and an improved BP control. It increases patients' engagement in the management of their condition, but professional time for supporting telemetry support and greater patient engagement can increase workloads and demand changes in service organisation. However, if these issues are overcome, BP telemonitoring could be an effective tool in the management of hypertension.

Acknowledgements Thanks are due to the practices and patients who participated in the study, to Lucy McCloughan and Mary Paterson for assistance with organisation, to Hillarie Higgins and Peter Fairbrother for assistance with data analysis. Paul Padfield and Sarah Wild were grant holders and contributed to the overall project. This study was overseen by the Telescot independent trial steering committee, Professor Sir Lewis Ritchie, Professor Ann-Louise Kinmonth and Professor Chris Griffiths.

Contributors BM, JH and CP designed the study. JH and BM led the research. JU collected the data and led the analysis. AS provided advice throughout the trial. All authors were involved in writing the paper.

Funding This study was funded by the BUPA Foundation (ref 748/G24) with additional support from the High Blood Pressure Foundation and NHS

Lothian. BM and $\mathrm{JH}$ were during the course of the study supported by the Scottish Chief Scientist Office. The funders had no role in the analysis, writing up of the results or decision to publish. The researchers were independent of the funders.

Competing interests None. 
Patient consent Obtained.

Ethics approval SE Scotland REC.

Provenance and peer review Not commissioned; externally peer reviewed.

Data sharing statement No additional data are available.

\section{REFERENCES}

1. Stroetmann KA, Kubitschke L, Robinson S, et al. How can telehealth help in the provision of integrated care? WHO Europe Health Systems and policy analysis policy briefing 13. Geneva: WHO, 2010.

2. Barlow J, Hendy J. Adopting integrated mainstream telecare services: lessons from the UK. Eurohealth 2009;15:8-9.

3. Pickering TG, Miller NH, Ogedegbe $\mathrm{G}$, et al. Call to action on use and reimbursement for home blood pressure monitoring: a joint scientific statement from the American Heart Association, American Society of Hypertension, and Preventive Cardiovascular Nurses Association. Hypertension 2008;52:10-29.

4. Okonofua EC, Simpson KN, Jesri A, et al. Therapeutic inertia is an impediment to achieving the healthy people 2010 blood pressure control goals. Hypertension 2006;47:345-51.

5. World Health Organisation. Prevention of cardiovascular disease: guidelines for assessment and management of total cardiovascular risk. Geneva: WHO, 2007.

6. National Institute for Health and Clinical Excellence. NICE guideline CG127: Management of hypertension in adults in Primary Care. London: NICE, 2011.

7. NHS. The Quality and Outcomes Framework, 2009/10. http://www.ic. nhs.uk/statistics-and-data-collections/audits-and-performance/ the-quality-and-outcomes-framework/the-quality-and-outcomesframework (accessed 30 Dec 2011).

8. Hodgkinson J, Mant J, Martin U, et al. Relative effectiveness of clinic and home blood pressure monitoring compared with ambulatory blood pressure monitoring in diagnosis of hypertension: systematic review. BMJ 2011;342:d3621.

9. Bobrie G, Chatellier G, Genes N, et al. Cardiovascular prognosis of 'masked hypertension' detected by blood pressure self-measurement in elderly treated hypertensive patients. JAMA 2004;291:1342-9.

10. Pickering TG. Measuring BP Pickering TG ed. Self-monitoring of blood pressure. In: Ambulatory monitoring and blood pressure variability (Part 1). London: Science Press, 1990, 8.3-8.5.

11. Parati G, Stergiou G. Self-measured and ambulatory blood pressure in assessing the white coat phenomenon. $J$ Hypertens 2003;21:677-82.

12. Lambert-Kerzner A, Havranck E, Plomondon ME, et al. Patients perspectives of a multifaceted intervention with a focus on technology: a qualitative analysis. Circ Cardiovasc Qual Outcomes 2010;3:668-74.

13. Jones MI, Greenfield SG, Bray EP, et al. Patients' experiences of self-monitoring blood pressure and self-titration of medication: the TASMINH2 trial qualitative study. Br J GenPract 2012;62:e135-42.

14. Ovaisi S, Ibison J, Leontowitsch M, et al. Stroke patients' perceptions of home blood pressure monitoring. Br J Gen Pract 2011 doi .3399/bjgp11X59389310
15. Abdullah A, Othman S. The influence of self-owned home blood pressure monitoring (HBPM) on primary care with hypertension: a qualitative study. BMC Fam Pract 2011;12:143.

16. McKinstry B, Hanley J, Wild S, et al. Telemonitoring-based service redesign for the management of uncontrolled hypertension (HITS): a multi-centre randomised controlled trial. In press.

17. Bray EP, Holder R, Mant J, et al. Does self-monitoring reduce blood pressure? Meta-analysis with meta-regression of randomised controlled trials. Ann Med 2010;42:371-86.

18. McManus RJ, Mant J, Bray EP, et al. Telemonitoring and self-management in the control of hypertension (TASMINH2): a randomised controlled trial. Lancet 2010;376:163-72.

19. Bosworth HB, Powers BJ, Olsen MK, et al. Can home blood pressure management improve blood pressure control: Results from a randomized controlled trial. Arch Intern Med 2011;171:1173-80.

20. Craig N, Dieppe P, Macintyre S, et al. Developing and evaluating complex interventions: the new Medical Research Council Guidance. BMJ 2008;337:a1655

21. Plsek PE, Greenhaulgh T. The challenge of complexity in health care. BMJ 2001;323:625.

22. Pawson R, Tilley N. Realistic evaluation. London: Sage, 1997.

23. Sandelowski M. Whatever happened to qualitative description? Res Nurs Health 2000;23:334-40.

24. Thorne S, Kirkham SR, MacDonald-Emes J. Interpretive description: a non-categorical qualitative alternative for developing nursing knowledge. Res Nurs Health 1997;20:169-77.

25. ISD Scotland: Practices and their populations http://www. isdscotland.org/Health-Topics/General-Practice/ Practices-and-Their-Populations/ (accessed 30 Jan 2012).

26. Bostock Y, Hanley J, McGown D, et al. The acceptability to patients and professionals of remote blood pressure monitoring using mobile phones. Health Care Res Dev 2009;10:299-308.

27. May C. A rational model for assessing and evaluating complex interventions in health care. BMC Health Ser Res 2006;6:86.

28. Ure J, Pinnock $\mathrm{H}$, Hanley $\mathrm{J}$, et al. Piloting tele-monitoring in COPD: a mixed methods exploration of issues in design and implementation. Prim Care Respir J 2012;21:57-64.

29. Fairbrother P, Ure J, Hanley J, et al. B on behalf of the TELESCOT programme team. Telemonitoring for chronic heart failure: the views of patients and healthcare professionals-a qualitative study. J Clin Nurs in press.

30. Mair F, May C, O'Donnell C, et al. Factors that promote or inhibit the implementation of e-health systems: an explanatory systematic review. Bull World Health Organ 2012;90:357-64.

31. May C, Harrison R, Finch T, et al. (2003) Understanding the normalisation of telemedicine services through qualitative evaluation. J Am Med Inform Assoc 2003;10:596-604.

32. McCant F, McCoy G, Grubber J, et al. Feasibility of blood pressure telemonitoring in patients with poor blood pressure control. $J$ Telemed Telecare 2009;15:281-5.

33. Scottish Executive Health Department. Guidance for nurse independent prescribers and for community practitioner nurse prescribers in Scotland. A guide for implementation. Edinburgh, SEHD, 2006.

34. ISD Scotland. Nurse Prescribing. http://www.isdscotlandarchive.scot nhs.uk/isd/2232.html (accessed 30 Jan 2012) 